TRANSACTIONS OF THE

AMERICAN MATHEMATICAL SOCIETY

Volume 352 , Number 9 , Pages 4195-4205

S 0002-9947(00)02655-6

Article electronically published on May 23, 2000

\title{
RESONANCE PROBLEMS WITH RESPECT TO THE FUČÍK SPECTRUM
}

\author{
MARTIN SCHECHTER
}

\begin{abstract}
We study semilinear boundary value problems which have asymptotic resonance with respect to the linear part. The difficulties for Fučík resonance problems are compounded by the fact that there is no eigenspace with which to work. The present paper uses new linking theorems which can deal with the sets required to obtain critical points.
\end{abstract}

\section{INTRODUCTION}

Let $\Omega$ be a bounded domain in $\mathbf{R}^{n}$, and let $A \geq \lambda_{0}>0$ be a selfadjoint operator or $L^{2}(\Omega)$ with compact resolvent and eigenvalues $0<\lambda_{0}<\lambda_{1}<\cdots<\lambda_{k}<\cdots$. If $f(x, t)$ is a Carathéodory function on $\Omega \times \mathbf{R}$, then the semilinear problem

$$
A u=f(x, u), \quad u \in D(A)
$$

is said to have asymptotic resonance at infinity if

$$
f(x, t) / t \rightarrow \lambda_{k} \text { as }|t| \rightarrow \infty,
$$

where $\lambda_{k}$ is one of the eigenvalues of $A$. Interest in resolvent problems began with the pioneering work of Landesman and Lazer [LL] and has continued until the present because they are more difficult to solve than nonresonant problems. The reason is that for $|u(x)|$ large, equation (1.1) approximates the eigenvalue problem

$$
A u=\lambda_{k} u
$$

with its inherent instabilities. Even if (1.2) does not occur, but

$$
\begin{aligned}
f(x, t) / t & \rightarrow a \text { as } t \rightarrow-\infty \\
& \rightarrow b \text { as } t \rightarrow+\infty ;
\end{aligned}
$$

one encounters the same difficulties if

$$
A u=b u^{+}-a u^{-}, \quad u^{ \pm}=\max \{ \pm u, 0\}
$$

has a nontrivial solution. In fact, the difficulties are compounded because there is no eigenspace that one can deal with in this case. These difficulties were recognized by Dancer $[\mathrm{D}]$ and Fučík $[\mathrm{F}]$ who studied the Dirichlet problem for second order ordinary differential equations. The Fučík spectrum $\Sigma$ of $A$ is defined to be the set of those points $(a, b) \in \mathbf{R}^{2}$ such that (1.5) has a nontrivial solution. For the case he considered, Fučík showed that $\Sigma$ consists of curves emanating from the points $\left(\lambda_{k}, \lambda_{k}\right)$ in the plane with one or two curves coming from each of these points.

Received by the editors August 20, 1996 and, in revised form, February 1, 1998.

2000 Mathematics Subject Classification. Primary 35J65, 58E05, 47J30, 49J40.

Research supported in part by an NSF grant.

(C)2000 American Mathematical Society 
In particular, $\Sigma$ has no interior points and exhibits the same instabilities as the eigenvalues. Most of the work to date concerning the situation in which the limits (1.4) exist stipulates that the point $(a, b)$ not be in the Fučík spectrum. The only exception of which the author is aware is the work of Costa-Cuesta [CC] in which $(a, b)$ is on a curve of $\Sigma$ passing through the eigenvalue $\left(\lambda_{1}, \lambda_{1}\right)$ for the Dirichlet problem for a second order partial differential equation. For the periodic problem in one-dimensions, they allow $(a, b)$ to be on any of the curves of $\Sigma$.

For the Dirichlet problem involving the Laplacian, several authors have shown that curves in $\Sigma$ exist locally in the neighborhood of the points $\left(\lambda_{k}, \lambda_{k}\right)$ (cf., e.g., $[\mathrm{F},[\mathrm{C}],[\mathrm{CG}], \mathrm{D}], \mathrm{GK}], \mathrm{L},[\mathrm{LM}], \mathrm{M}],[\mathrm{R}])$. In $[\mathrm{S} 1$ we showed that in the square $Q_{\ell}=\left\{(a, b) \in \mathbf{R}^{2}: \lambda_{\ell-1}<a, b<\lambda_{\ell+1}\right\}$ there are two curves $C_{\ell, 1}, C_{\ell, 2}$ (possibly coinciding) in $\Sigma$ which are decreasing and pass through the point $\left(\lambda_{\ell}, \lambda_{\ell}\right)$. All points in the square except those on the curves and possibly those between the curves are not in $\Sigma$. If $C_{\ell, 1}$ denotes the "lower" curve, all points in $Q_{\ell}$ below $C_{\ell, 1}$ are free of $\Sigma$. Similarly, if $C_{\ell, 2}$ denotes the "upper" curve, all points in $Q_{\ell}$ above $C_{\ell, 2}$ are not in $\Sigma$. Concerning the points between the curves, it is not known in general what their status is. However, it was shown in GK that they are not in $\Sigma$ if $\lambda_{\ell}$ is a simple eigenvalue. C. and W. Margulies have constructed examples in which there are numerous curves in $\Sigma$ emanating from the point $\left(\lambda_{\ell}, \lambda_{\ell}\right)$. Of course, such curves must lie between $C_{\ell, 1}$ and $C_{\ell, 2}$.

In the present paper we shall study resonance problems with respect to the Fučík spectrum. We shall allow $(a, b)$ to be on either of the curves $C_{\ell, j}$ in $Q_{\ell}$. We do not consider the case when $(a, b)$ is a point between the curves (when there are points in $\Sigma$ in that region). We have

Theorem 1.1. Let $f(x, t)$ be a Carathéodory function such that

$$
|f(x, t)| \leq C(|t|+1), \quad x \in \Omega, t \in \mathbf{R},
$$

and (1.4) holds with $(a, b) \in C_{\ell, 1}$. Assume that

$$
\lambda_{\ell-1} t^{2} \leq 2 F(x, t)+W_{1}(x), \quad x \in \Omega, t \in \mathbf{R},
$$

and

$$
H(x, t) \leq W_{0}(x), \quad x \in \Omega, t \in \mathbf{R},
$$

where

$$
F(x, t):=\int_{0}^{t} f(x, s) d s
$$

and

$$
H(x, t):=2 F(x, t)-t f(x, t) .
$$

Assume further that

$$
H(x, t) \rightarrow H_{ \pm}(x) \text { a.e. as } t \rightarrow \pm \infty,
$$

and

$$
\int_{u>0} H_{+}(x) d x+\int_{u<0} H_{-}(x) d x+\int_{u=0} W_{0}(x) d x<-B_{1}=-\int_{\Omega} W_{1}(x) d x
$$

whenever $u$ is a nontrivial solution of (1.5). Then (1.1) has a solution. 
Theorem 1.2. If (1.6) and (1.4) hold with $(a, b) \in C_{\ell, 2}$, assume that

$$
\begin{gathered}
2 F(x, t) \leq \lambda_{\ell+1} t^{2}+W_{1}(x), \quad x \in \Omega, t \in \mathbf{R}, \\
H(x, t) \geq-W_{0}(x), \quad x \in \Omega, t \in \mathbf{R}
\end{gathered}
$$

and

$$
\int_{u>0} H_{+}(x) d x+\int_{u<0} H_{-}(x) d x-\int_{u=0} W_{0}(x) d x>B_{1}
$$

for all nontrivial solutions $u$ of (1.5). Then (1.1) has a solution.

Note that (1.12) is automatically satisfied if

$$
H(x, t) \rightarrow-\infty \text { a.e. as }|t| \rightarrow \infty,
$$

and (1.15) is automatically satisfied if

$$
H(x, t) \rightarrow+\infty \text { a.e. as }|t| \rightarrow \infty .
$$

The proofs of Theorems 1.1 and 1.2 are given in Section 4 after preliminary work in Sections 2 and 3.

\section{LINKING}

In [ST], K. Tintarev and the author introduced a new concept of linking which allowed more extensive and far reaching mountain pass and saddle point theorems. We now present a refined version here. Let $E$ be a Banach space and let $\Phi$ be the set of all continuous maps $\Gamma=\Gamma(t)$ from $E \times[0,1]$ to $E$ such that

1. $\Gamma(0)=I$, the identity on $E$.

2. For each $t \in[0,1), \Gamma(t)$ is a homeomorphism of $E$ onto $E$ and $\Gamma^{-1}(t) \in$ $C(E \times[0,1), E)$.

3. $\Gamma(1) E$ is a single point of $E$ and $\Gamma(t) A$ converges uniformly to $\Gamma(1) E$ as $t \rightarrow 1$ for each bounded set $A \subset E$.

4. For each $t_{0} \in[0,1)$ and each bounded set $A \subset E$

$$
\sup _{\substack{0 \leq t \leq t_{0} \\ u \in A}}\left\{\|\Gamma(t) u\|+\left\|\Gamma^{-1}(t) u\right\|\right\}<\infty .
$$

Definition. For $A, B \subset E$ we say that $A$ links $B$ if

a) $A \cap B=\phi$;

b) for each $\Gamma \in \Phi$ there is a $t \in(0,1]$ such that

$$
\Gamma(t) A \cap B \neq \phi .
$$

We have

Proposition 2.1. If $A, B \subset E$ are closed and bounded, $E \backslash A$ is pathwise connected and $A$ links $B$, then $B$ links $A$.

Proposition 2.2. If $F \in C\left(E, \mathbf{R}^{n}\right)$ and $Q \subset E$ is such that $F_{0}=\left.F\right|_{Q}$ is a homeomorphism of $Q$ onto the closure of a bounded open subset $\Omega$ of $\mathbf{R}^{n}$, then $\partial Q=F_{0}^{-1}(\partial \Omega)$ links $F^{-1}(p)$ for each $p \in \Omega$. 
Theorem 2.3. Let $G$ be a $C^{1}$ functional on $E$, and let $A, B$ be subsets of $E$ such that $A$ is bounded and links B. Assume

$$
\begin{gathered}
a_{0}:=\sup _{A} G \leq b_{0}:=\inf _{B} G \\
a:=\inf _{\Gamma \in \Phi} \sup _{\substack{0 \leq s \leq 1 \\
u \in A}} G(\Gamma(s) u)<\infty .
\end{gathered}
$$

Let $\psi(t)$ be a positive nonincreasing function on $(0, \infty)$ such that

$$
\int_{1}^{\infty} \psi(r) d r=\infty .
$$

Then there is a sequence $\left\{u_{k}\right\} \subset E$ such that

$$
G\left(u_{k}\right) \rightarrow a, \quad G^{\prime}\left(u_{k}\right) / \psi\left(\left\|u_{k}\right\|\right) \rightarrow 0 .
$$

Corollary 2.4. Under the hypotheses of Theorem 2.3 there is a sequence $\left\{u_{k}\right\} \subset E$ such that

$$
G\left(u_{k}\right) \rightarrow a, \quad\left(1+\left\|u_{k}\right\|\right) G^{\prime}\left(u_{k}\right) \rightarrow 0 .
$$

Proposition 2.5. Let $H$ be a homeomorphism of $E$ onto itself such that $H$ and $H^{-1}$ map bounded sets into bounded sets. If $A, B \subset E$ and $A$ links $B$, then $H A$ links $H B$.

Proposition 2.6. Let $M, N$ be closed subspaces of $E$ such that

$$
\operatorname{dim} N<\infty
$$

and

$$
E=M \oplus N
$$

Let $w_{0} \neq 0$ be an element of $M$, and take

$$
A=\{v \in N:\|v\| \leq R\} \cup\left\{s w_{0}+v: s \geq 0, v \in N,\left\|s w_{0}+v\right\|=R\right\}
$$

and

$$
B=M \cap \partial B_{\delta}
$$

with $0<\delta<R$, where $B_{R}=\{u \in E:\|u\|<R\}$. Then $A$ and $B$ link each other.

Proposition 2.7. If (2.8) and (2.9) hold, then $M \cap \partial B_{R}$ links $N$ for each $R>0$.

Proofs of the statements of this section can be found in [S2].

\section{THE CURVES}

In this section we shall describe the curves $C_{\ell, 1}$ and $C_{\ell, 2}$ and discuss some of their properties. For each fixed positive integer $\ell$ we let $N_{\ell}$ denote the subspace of $E$ spanned by the eigenfunctions corresponding to $\lambda_{0}, \cdots, \lambda_{\ell}$, and we let $M_{\ell}=$ $N_{\ell}^{\perp} \cap D$. Then $D=M_{\ell} \oplus N_{\ell}$. For $a, b \in \mathbf{R}$ we define

$$
\begin{gathered}
I(u, a, b)=(A u, u)-a\left\|u^{-}\right\|^{2}-b\left\|u^{+}\right\|^{2}, \quad u \in D, \\
M_{\ell}(a, b)=\inf _{\substack{w \in M_{\ell} \\
\|w\|=1}} \sup _{v \in N_{\ell}} I(v+w, a, b), \\
m_{\ell}(a, b)=\sup _{\substack{v \in N_{\ell} \\
\|v\|=1}} \inf _{w \in M_{\ell}} I(v+w, a, b),
\end{gathered}
$$




$$
\begin{aligned}
& \nu_{\ell}(a)=\sup \left\{b: M_{\ell}(a, b) \geq 0\right\}, \\
& \mu_{\ell}(a)=\inf \left\{b: m_{\ell}(a, b) \leq 0\right\} .
\end{aligned}
$$

We have

Lemma 3.1 ([S1]). In the square $Q_{\ell}$ the functions $\mu_{\ell}(a), \nu_{\ell-1}(a)$ have the following properties:

a) $\mu_{\ell}\left(\lambda_{\ell}\right)=\nu_{\ell-1}\left(\lambda_{\ell}\right)=\lambda_{\ell}$,

b) $\mu_{\ell}(a), \nu_{\ell-1}(a)$ are continuous and strictly decreasing,

c) $\left(a, \mu_{\ell}(a)\right)$ and $\left(a, \nu_{\ell-1}(a)\right)$ are in $\Sigma$.

d) If $(a, b) \in Q_{\ell}$ and $b>\mu_{\ell}(a)$, then $(a, b) \notin \Sigma$.

e) If $(a, b) \in Q_{\ell}$ and $b<\nu_{\ell-1}(a)$, then $(a, b) \notin \Sigma$.

It follows from Lemma 3.1 that $\nu_{\ell-1}(a) \leq \mu_{\ell}(a)$ in $Q_{\ell}$. If $\nu_{\ell-1}(a)<\mu_{\ell}(a)$, this lemma does not cover those points in $Q_{\ell}$ for which

$$
\nu_{\ell-1}(a)<b<\mu_{\ell}(a) \text {. }
$$

We let $C_{\ell, 1}$ be the lower curve $b=\nu_{\ell-1}(a)$ and we take $C_{\ell, 2}$ to be the upper curve $b=\mu_{\ell}(a)$. We shall need

Lemma 3.2 ([S1]). If $(a, b) \in Q_{\ell}, w \in M_{\ell-1}, v_{1}, v_{2} \in N_{\ell-1}$ and

$$
I^{\prime}\left(w+v_{j}, a, b\right) \perp N_{\ell-1}, \quad j=1,2,
$$

then $v_{1}=v_{2}$.

Lemma 3.3 ([S1] $)$. If $(a, b) \in Q_{\ell}, v \in N_{\ell}, w_{1}, w_{2} \in M_{\ell}$ and

$$
I^{\prime}\left(v+w_{j}, a, b\right) \perp M_{\ell}, \quad j=1,2,
$$

then $w_{1}=w_{2}$.

Lemma 3.4 ([S1]). If $(a, b) \in Q_{\ell}$, then for each $w \in M_{\ell-1}$ there is a $v_{0} \in N_{\ell-1}$ such that

$$
I\left(w+v_{0}, a, b\right)=\sup _{v \in N_{\ell-1}} I(w+v, a, b) .
$$

Lemma 3.5 ([S1]). If $(a, b) \in Q_{\ell}$, then for each $v \in N_{\ell}$ there is a $w_{0} \in M_{\ell}$ such that

$$
I\left(v+w_{0}, a, b\right)=\inf _{w \in M_{\ell}} I(v+w, a, b) .
$$

Lemma 3.6. If $(a, b) \in C_{\ell, 1}$, then

$$
I(u, a, b) \geq 0, \quad u \in S_{\ell, 1},
$$

where

$$
S_{\ell, 1}=\left\{u \in E: I^{\prime}(u, a, b) \perp N_{\ell-1}\right\} .
$$

Proof. If $(a, b) \in C_{\ell, 1}$, then $b=\nu_{\ell-1}(a)$. Thus by (3.4)

$$
M_{\ell-1}(a, b)=0 .
$$

This implies, by (3.2), that

$$
\sup _{v \in N_{\ell-1}} I(v+w, a, b) \geq 0, \quad w \in M_{\ell-1} .
$$


Let $u_{0}$ be any element in $S_{\ell, 1}$. Then $u_{0}=w_{0}+v_{0}, w_{0} \in M_{\ell-1}, v_{0} \in N_{\ell-1}$ and

$$
I^{\prime}\left(w_{0}+v_{0}, a, b\right) \perp N_{\ell-1}
$$

by (3.11). In view of Lemma 3.4 there is a $v_{1} \in N_{\ell-1}$ such that

$$
I\left(w_{0}+v_{1}, a, b\right)=\sup _{v \in N_{\ell-1}} I\left(w_{0}+v, a, b\right) .
$$

From this and (3.13) it follows that

$$
I^{\prime}\left(w_{0}+v_{1}, a, b\right) \perp N_{\ell-1}
$$

and

$$
I\left(w_{0}+v_{1}, a, b\right) \geq 0 .
$$

But (3.14) and (3.16) imply via Lemma 3.2 that $v_{1}=v_{0}$. Thus $I\left(u_{0}, a, b\right) \geq 0$, and the lemma is proved.

Lemma 3.7. If $(a, b) \in C_{\ell, 2}$, then

$$
I(u, a, b) \leq 0, \quad u \in S_{\ell, 2},
$$

where

$$
S_{\ell, 2}=\left\{u \in E: I^{\prime}(u, a, b) \perp M_{\ell}\right\}
$$

Proof. If $(a, b) \in C_{\ell, 2}$, then $b=\mu_{\ell}(a)$. Consequently, by (3.5)

$$
m_{\ell}(a, b)=0 .
$$

By (3.3) this implies

$$
\inf _{w \in M_{\ell}} I(v+w, a, b) \leq 0, \quad v \in N_{\ell}
$$

Let $u_{0}$ be any element in $S_{\ell, 2}$, and write $u_{0}=v_{0}+w_{0}$ with $v_{0} \in N_{\ell}, w_{0} \in M_{\ell}$. Then

$$
I^{\prime}\left(v_{0}+w_{0}, a, b\right) \perp M_{\ell} .
$$

By Lemma 3.5 there is a $w_{1} \in M_{\ell}$ such that

$$
I\left(v_{0}+w_{1}, a, b\right)=\inf _{w \in M_{\ell}} I\left(v_{0}+w, a, b\right) .
$$

Consequently,

$$
I^{\prime}\left(v_{0}+w_{1}, a, b\right) \perp M_{\ell}
$$

and

$$
I\left(v_{0}+w_{1}, a, b\right) \leq 0 .
$$

If we now apply Lemma 3.3 we see that $w_{1}=w_{0}$. Thus $I\left(u_{0}, a, b\right) \leq 0$, and the proof is complete.

Lemma 3.8. $N_{\ell-1} \cap \partial B_{R}$ links $S_{\ell, 1}$ for each $R>0$.

Proof. We suppress the subscript $\ell-1$. Let $P$ be the orthogonal projection of $D$ onto $N$, and define

$$
F(u)=P I^{\prime}(u), \quad u \in D .
$$

I claim that the restriction $F_{0}$ of $F$ to $N$ is a homeomorphism of $N$ onto $N$. If this is so, then the image of $N \cap \bar{B}_{R}$ under $F_{0}$ is the closure of a bounded open set. Moreover, this open set contains the point 0 since $F_{0}(0)=0$, and 0 is an interior 
point of $N \cap B_{R}$. Proposition 2.2 can then be used to show that $N \cap \partial B_{R}$ links $F^{-1}(0)=S_{\ell, 1}$. Clearly, $F_{0}$ is a continuous map of $N$ into itself. It is surjective. To see this let $h$ be any element of $N$ and take

$$
G_{0}(v)=I(v, a, b)-(h, v) .
$$

We note that

$$
G_{0}(v) \rightarrow-\infty \text { as }\|v\| \rightarrow \infty
$$

Assuming this for the moment, we see that $G_{0}(v)$ has a maximum on $N$. At the point of maximum we have $G_{0}^{\prime}(v) \perp N$ producing a solution of

$$
P I^{\prime}(v, a, b)=h .
$$

Moreover, if

$$
P I^{\prime}\left(v_{0}, a, b\right)=h_{0}, P I^{\prime}\left(v_{1}, a, b\right)=h_{1}
$$

then

$$
(h, v)=(A v, u)+a\left(v_{1}^{-}-v_{0}^{-}, v\right)-b\left(v_{1}^{+}-v_{0}^{+}, v\right),
$$

where $h=h_{1}-h_{0}, v=v_{1}-v_{0}$. Thus

$$
\begin{gathered}
\left(b-\lambda_{\ell-1}\right)\left(v_{1}^{+}-v_{0}^{+}, v\right)-\left(a-\lambda_{\ell-1}\right)\left(v_{1}^{-}-v_{0}^{-}, v\right) \\
+\left[\lambda_{\ell-1}\|v\|^{2}-(A v, v)\right]=-(h, v) .
\end{gathered}
$$

Since $a, b>\lambda_{\ell-1}$, the left hand side of (3.30) is positive for all $v \not \equiv 0$. Consequently, there is a $\delta>0$ such that

$$
\delta\|v\|^{2} \leq\|h\|\|v\|,
$$

showing not only that $F_{0}$ is injective but that $F_{0}^{-1}$ is continuous. To prove (3.27), let $\left\{v_{k}\right\} \subset N$ be a sequence such that $\rho_{k}^{2}=\left(A v_{k}, v_{k}\right) \rightarrow \infty$, and let $\tilde{v}_{k}=v_{k} / \rho_{k}$. Then $\left\|\tilde{v}_{k}\right\|_{D}^{2}=\left(A \tilde{v}_{k}, \tilde{v}_{k}\right)=1$, and there is a subsequence such that $\tilde{v}_{k} \rightarrow \tilde{v}$ in $D$. We have

$$
\begin{aligned}
G_{0}\left(v_{k}\right) / \rho_{k}^{2} & =I\left(\tilde{v}_{k}, a, b\right)-\left(h, \tilde{v}_{k}\right) / \rho_{k} \rightarrow I(\tilde{v}, a, b) \\
& =\left[\|\tilde{v}\|_{D}^{2}-\lambda_{\ell-1}\|\tilde{v}\|^{2}\right]+\left(\lambda_{\ell-1}-a\right)\left\|\tilde{v}^{-}\right\|^{2}+\left(\lambda_{\ell-1}-b\right)\left\|\tilde{v}^{+}\right\|^{2} .
\end{aligned}
$$

Since $\|\tilde{v}\|_{D}=1$, the right hand side of (3.32) is negative. This gives (3.27) and completes the proof of the lemma.

\section{EXISTENCE}

In this section we give the proofs of Theorems 1.1 and 1.2. Let

$$
p(x, t)=f(x, t)+a t^{-}-b t^{+}, \quad t^{ \pm}=\max \{ \pm t, 0\}
$$

and

$$
P(x, t)=\int_{0}^{t} p(x, s) d s .
$$

Under hypothesis (1.6) it is readily checked that the functional

$$
G(u)=\|u\|_{D}^{2}-2 \int_{\Omega} F(x, u) d x=I(u, a, b)-2 \int_{\Omega} P(x, u) d x
$$


is in $C^{1}$ on $D$ with

$$
\left(G^{\prime}(u), v\right)=2(u, v)_{D}-2(f(u), v)=\left(I^{\prime}(u, a, b), v\right)-2(p(u), v)
$$

and

$$
\left(I^{\prime}(u, a, b), v\right) / 2=(u, v)_{D}+a\left(u^{-}, v\right)-b\left(u^{+}, v\right),
$$

and we write $f(u), p(u)$ in place of $f(x, u), p(x, u)$, respectively. From (1.4) and (4.1) we see that

$$
p(x, t) / t \rightarrow 0, \quad 2 P(x, t) / t^{2} \rightarrow 0 \text { as }|t| \rightarrow \infty .
$$

This and the fact that

$$
\partial\left(F / t^{2}\right) / \partial t=\partial\left(P / t^{2}\right) / \partial t=-t^{-3} H
$$

imply that

$$
\begin{aligned}
P(x, t) & =t^{2} \int_{t}^{\infty} s^{-3} H(x, s) d s, \quad t>0, \\
& =-t^{2} \int_{-\infty}^{t} s^{-3} H(x, s) d s, \quad t<0 .
\end{aligned}
$$

Proof of Theorem 1.1. By (1.8) and (4.8) we have

$$
2 P(x, t) \leq W_{0}(x), \quad x \in \Omega, t \in \mathbf{R} .
$$

In view of Lemma 3.6 this implies

$$
G(u) \geq-2 \int_{\Omega} P(x, u) d x \geq-B_{0}=-\int_{\Omega} W_{0}(x) d x, \quad u \in S_{\ell, 1} .
$$

I claim that

$$
G(v) \rightarrow-\infty \text { as }\|v\| \rightarrow \infty, \quad v \in N_{\ell-1} .
$$

Assume this for the moment. Then there is an $R>0$ sufficiently large that (2.3) holds with $A=N_{\ell-1} \cap \partial B_{R}$ and $B=S_{\ell, 1}$. Moreover, for this choice of $A$ and $B$, $A$ links $B$ by Lemma 3.8. We can now apply Theorem 2.3 to conclude that there is a sequence $\left\{u_{k}\right\} \subset D$ such that

$$
G\left(u_{k}\right) \rightarrow c, \quad\left(1+\left\|u_{k}\right\|_{D}\right) G^{\prime}\left(u_{k}\right) \rightarrow 0,
$$

and from (4.3) we readily estimate $c$ by

$$
-B_{0} \leq c \leq B_{1}
$$

From (4.12) we find

$$
\begin{gathered}
I\left(u_{k}, a, b\right)-2 \int_{\Omega} P\left(x, u_{k}\right) d x \rightarrow c, \\
I\left(u_{k}, a, b\right)-\left(p\left(u_{k}\right), u_{k}\right) \rightarrow 0, \\
\left(I^{\prime}\left(u_{k}, a, b\right), v\right)-2\left(p\left(u_{k}\right), v\right) \rightarrow 0, \quad v \in D .
\end{gathered}
$$

Assume that

$$
\rho_{k}=\left\|u_{k}\right\|_{D} \rightarrow \infty
$$


and let $\tilde{u}_{k}=u_{k} / \rho_{k}$. Then $\left\|\tilde{u}_{k}\right\|_{D}=1$. Thus there is a renamed subsequence such that $\tilde{u}_{k} \rightarrow \tilde{u}$ weakly in $D$, strongly in $L^{2}(\Omega)$ and a.e. in $\Omega$. As a consequence, (4.6) and (4.15) imply

$$
1=a\left\|\tilde{u}^{-}\right\|^{2}+b\left\|\tilde{u}^{+}\right\|^{2} .
$$

This shows that $\tilde{u} \not \equiv 0$. Moreover, (4.16) implies

$$
I^{\prime}(\tilde{u}, a, b)=0 .
$$

Thus $\tilde{u}$ is a solution of (1.5) and satisfies

$$
I(\tilde{u}, a, b)=0 .
$$

If we combine this with (4.18), we see that $\|\tilde{u}\|_{D}=1$. Consequently, $\tilde{u}_{k} \rightarrow \tilde{u}$ strongly in $D$. Combining (4.14) and (4.15) we obtain

$$
\int_{\Omega} H\left(x, u_{k}\right) d x \rightarrow-c .
$$

Let $\Omega_{ \pm}=\{x \in \Omega: \pm \tilde{u}(x)>0\}, \Omega_{0}=\{x \in \Omega: \tilde{u}(x)=0\}$. Then $u_{k}(x) \rightarrow \pm \infty$ for $x \in \Omega_{ \pm}$. By (1.8) and (1.12)

$$
-c=\lim \int_{\Omega} H\left(x, u_{k}\right) d x \leq \int_{\Omega_{+}} H_{+}(x) d x+\int_{\Omega_{-}} H_{-}(x) d x+\int_{\Omega_{0}} W_{0}(x) d x<-B_{1}
$$

contradicting (4.13). Thus (4.17) does not hold. Once we know that the sequence $\left\{u_{k}\right\}$ is bounded in $D$, we can use standard techniques to obtain a solution of

$$
G(u)=c, \quad-B_{0} \leq c \leq B_{1}, \quad G^{\prime}(u)=0,
$$

which is a solution of (1.1).

It thus remains to prove (4.11). Let $\left\{v_{k}\right\} \subset N_{\ell-1}$ be a sequence such that $\rho_{k}=\left\|v_{k}\right\|_{D} \rightarrow \infty$. Let $\tilde{v}_{k}=v_{k} / \rho_{k}$. Then $\left\|\tilde{v}_{k}\right\|_{D}=1$, and there is a renamed subsequence such that $\tilde{v}_{k} \rightarrow \tilde{v}$ in $D$ and a.e. in $\Omega$. Since

$$
|F(x, t)| \leq C\left(t^{2}+|t|\right)
$$

by (1.6), we have

$$
\left|F\left(x, v_{k}\right) / \rho_{k}^{2}\right| \leq C\left(\tilde{v}_{k}^{2}+\left|\tilde{v}_{k}\right| / \rho_{k}\right),
$$

and consequently,

$$
2 \int_{\Omega} F\left(x, v_{k}\right) d x / \rho_{k}^{2} \rightarrow a\left\|\tilde{v}^{-}\right\|^{2}+b\left\|\tilde{v}^{+}\right\|^{2}
$$

by (1.4). This means that

$$
\begin{aligned}
G\left(v_{k}\right) / \rho_{k}^{2} & =\left\|\tilde{v}_{k}\right\|_{D}^{2}-2 \int_{\Omega} F\left(x, v_{k}\right) d x / \rho_{k}^{2} \\
& \rightarrow\|\tilde{v}\|_{D}^{2}-a\left\|\tilde{v}^{-}\right\|^{2}-b\left\|\tilde{v}^{+}\right\|^{2} \\
& \leq\left(\lambda_{\ell-1}-a\right)\left\|\tilde{v}^{-}\right\|^{2}+\left(\lambda_{\ell-1}-b\right)\left\|\tilde{v}^{+}\right\|^{2} .
\end{aligned}
$$

Since both $a$ and $b$ are greater than $\lambda_{\ell-1}$, this will always be negative since $\tilde{v} \not \equiv 0$. Thus (4.11) holds, and the proof of Theorem 1.1 is complete. 
Proof of Theorem 1.2. By (1.14) and (4.8) we have

$$
-2 P(x, t) \leq W_{0}(x), \quad x \in \Omega, t \in \mathbf{R} .
$$

Consequently,

$$
G(u) \leq-2 \int_{\Omega} P(x, u) d x \leq B_{0}, \quad u \in S_{\ell, 2} .
$$

Moreover, I claim that

$$
G(w) \rightarrow \infty \text { as }\|w\| \rightarrow \infty, \quad w \in M_{\ell} .
$$

Assuming this, we note that there is an $R>0$ sufficiently large such that

$$
\sup _{B} G \leq \inf _{A} G
$$

where $A=M \cap \partial B_{R}$ and $B=S_{\ell, 2}$. This is not quite (2.3). To correct the situation, we let $G_{1}=-G$. Then (4.26) becomes

$$
\sup _{A} G_{1} \leq \inf _{B} G_{1} .
$$

We know from Proposition 2.7 that $A$ links $B$. Consequently, we may apply Theorem 2.3 to conclude that there is a sequence $\left\{u_{k}\right\} \subset D$ such that (4.12) holds with $G$ replaced by $G_{1}$. Now $c$ satisfies the estimates

$$
\inf _{B} G_{1} \leq c \leq \sup _{M \cap B_{R}} G_{1}
$$

or

$$
\inf _{M \cap B_{R}} G \leq-c \leq \sup _{B} G .
$$

By (1.13), (2.4), (4.3) and (4.24), we see that $c$ satisfies (4.13) as well. Thus for the given sequence, (4.12), (4.14)-(4.16) hold with $c$ replaced by $-c$, while $c$ satisfies (4.13). If we assume that (4.17) holds, we can reason as in the proof of Theorem 1.1 that there is a renamed subsequence of $\left\{\tilde{u}_{k}\right\}$ converging in $D$ to a function $\tilde{u} \in D$ and a.e. in $\Omega$. Moreover, the function $\tilde{u}$ satisfies (4.19) and (4.20). Also (4.21) holds with $c$ replaced by $-c$. But then (1.14) and (1.15) imply

$$
c=\lim \int_{\Omega} H\left(x, u_{k}\right) d x \geq \int_{\Omega_{+}} H_{+}(x) d x+\int_{\Omega_{-}} H_{-}(x) d x-\int_{\Omega_{0}} W_{0}(x) d x .
$$

This contradiction shows that the $\rho_{k}$ are bounded, and we can now employ standard techniques to obtain a solution of

$$
G(u)=c_{1}, \quad-B \leq c_{1} \leq B_{0}, \quad G^{\prime}(u)=0 .
$$

This produces the desired result.

It therefore remains only to prove (4.25). Let $\left\{w_{k}\right\} \subset M_{\ell}$ be a sequence such that $\rho_{k}=\left\|w_{k}\right\|_{D} \rightarrow \infty$. Let $\tilde{w}_{k}=w_{k} / \rho_{k}$. Then we have $\left\|\tilde{w}_{k}\right\|_{D}=1$, and there is a renamed subsequence such that $\tilde{w}_{k} \rightarrow \tilde{w}$ weakly in $D$, strongly in $L^{2}(\Omega)$ and a.e. in $\Omega$. Thus

$$
2 \int_{\Omega} F\left(x, w_{k}\right) d x / \rho_{k}^{2} \rightarrow a\left\|\tilde{w}^{-}\right\|^{2}+b\left\|\tilde{w}^{+}\right\|^{2} .
$$

Consequently,

$$
\begin{aligned}
G\left(w_{k}\right) / \rho_{k}^{2} \rightarrow & 1-a\left\|\tilde{w}^{-}\right\|^{2}-b\left\|\tilde{w}^{+}\right\|^{2} \\
& \geq 1-\|\tilde{w}\|_{D}^{2}+\left(\lambda_{\ell+1}-a\right)\left\|\tilde{w}^{-}\right\|^{2}+\left(\lambda_{\ell+1}-b\right)\left\|\tilde{w}^{+}\right\|^{2}
\end{aligned}
$$


which is positive since both $a$ and $b$ are less than $\lambda_{\ell+1}$. This completes the proof of the theorem.

\section{REFERENCES}

[C] N.P. Cac, On nontrivial solutions of a Dirichlet problem whose jumping nonlinearity crosses a multiple eigenvalue, J. Diff. Eq. 80(1989) 379-404. MR 90f:35077

[CC] D.G. Costa and M. Cuesta, Existence results for resonant perturbations of the Fucik spectrum, Topol. Methods Nonlinear Anal. 8 (1996) 295-314. MR 99c:35056

[CG] M. Cuesta and J.-P Gossez, A variational approach to nonresonance with respect to the Fučík spectrum, Nonlinear Anal. TMA, 19 (1992) 487-500. MR 93i:34032

[D] E. Dancer, On the Dirichlet problem for weakly nonlinear elliptic partial differential equations, Proc. Royal Soc. Edinburgh, 76A (1977) 283-300. MR 58:17506; MR 82i:35063

[F] S. Fučík, Boundary value problems with jumping nonlinearities, Časopis Pest. Mat. 101 (1976) 69-87. MR 56:5998

[dFG] D.G. de Figueiredo and J.-P Gossez, On the first curve of the Fučík spectrum, preprint.

[GK] T. Gallouet and O. Kavian, Resultats d'existence et de non-existence pour certains problemes demi lineaires a l'infini, Ann. Fac. Sci. Toulouse Math. 3 (1981) 201-246. MR 83m:35058

[K] P. Krejci, On solvability of equations of the 4th order with jumping nonlinearities, Cas. Pest. Mat. 108 (1983) 29-39. MR 84i:35018

[L] A.C. Lazer, Introduction to multiplicity theory for boundary value problems with asymmetric nonlinearities, Partial Differential Equations, F. Cardos at al., Eds., Lecture Notes in Mathematics, No. 1324, Springer, N.Y. 1988, pp. 137-165. MR 90c:35093

[LL] E.A. Landesman and A.C. Lazer, Nonlinear perturbations of linear elliptic boundary value problems at resonance, J. Math. Mech. 19 (1970) 609-623. MR 42:2171

[LM] A.C. Lazer and P.J. McKenna, Critical point theory and boundary value problems with nonlinearities crossing multiple eigenvalues, Comm. P.D.E., I, 10 (1985) 107-150, II, 11 (1986) 1653-1676. MR 88c:35063

[M] C. Magalhães, Semilinear elliptic problem with crossing of multiple eigenvalues, Comm. P.D.E. 15 (1990) 1265-1292. MR 91h:35127

[R] P.H. Rabinowitz, Minimax methods in critical point theory with applications to differential equations, Conf. Board of Math. Sci. Reg. Conf. Ser. in Math., No. 65, Amer. Math. Soc., 1986. MR 87j:58024

[R] B. Ruf, On nonlinear elliptic problems with jumping nonlinearities, Ann. Mat. Pure Appl. 128 (1981) 133-151. MR 83e:35054

[S] M. Schechter, Asymptotically linear elliptic boundary value problems, Monatshefte fur Math. 117 (1994) 121-137. MR 95c:35108

[S1] M. Schechter, The Fučík spectrum, Indiana Univ. Math. J., 43 (1994) 1139-1157. MR 96c:35063

[S2] M. Schechter, New linking theorems, Rend. Sem. Mat. Univ. Padova 99 (1998), 255-269. MR 99h:58035

[ST] M. Schechter and K. Tintarev, Pairs of critical points produced by linking subsets with applications to semilinear elliptic problems, Bull. Soc. Math. Belg. 44 (1992) 249-261. MR 95k:58033

Department of Mathematics, University of California, Irvine, California 92697-3875

E-mail address: mschecht@math.uci.edu

E-mail address: mschecht@juno.com

$U R L:$ http://www.math.uci.edu/mschecht.html 